\title{
Effectiveness of Sorafenib in Patients with Transcatheter Arterial Chemoembolization (TACE) Refractory and Intermediate-Stage Hepatocellular Carcinoma
}

\author{
Tadaaki Arizumi Kazuomi Ueshima Tomohiro Minami \\ Masashi Kono Hirokazu Chishina Masahiro Takita Satoshi Kitai \\ Tatsuo Inoue Norihisa Yada Satoru Hagiwara Yasunori Minami \\ Toshiharu Sakurai Naoshi Nishida Masatoshi Kudo \\ Department of Gastroenterology and Hepatology, Kinki University Faculty of Medicine, Osaka, Japan
}

\author{
Key Words \\ Child-Pugh score $\cdot$ Hepatocellular carcinoma · Overall survival · Sorafenib · \\ Transarterial chemoembolization
}

\begin{abstract}
Background and Aims: Patients with intermediate-stage hepatocellular carcinoma (HCC) refractory to transcatheter arterial chemoembolization (TACE) are considered to be candidates for sorafenib. The aim of this study was to evaluate the superiority of conversion of treatment to sorafenib on overall survival (OS) for cases refractory to TACE. Methods: This was a retrospective cohort study carried out on 497 patients with HCC who were treated with TACE therapy at our hospital between January 2008 and December 2013. Fifty-six patients were diagnosed as refractory to TACE during their clinical course and they were divided into two cohorts, (1) those who switched from TACE to sorafenib and (2) those who continued TACE. The overall survival (OS) after the time of being refractory to TACE was evaluated between the two groups. Results: After refractoriness to TACE therapy was confirmed, 24 patients continued with TACE (TACE-group) and 32 patients underwent treatment conversion to sorafenib (sorafenib-group). The median OS was 24.7 months in the sorafenib-group and 13.6 months
\end{abstract}

Masatoshi Kudo, MD, PhD

Department of Gastroenterology and Hepatology,

Kinki University Faculty of Medicine

377-2 Ohno-Higashi, Osaka-Sayama, Osaka 589-8511 (Japan)

Tel.+81 72366 0221, E-Mail m-kudo@med.kindai.ac.jp

KARGER 125 
in the TACE-group $(p=0.002)$. Conclusions: Conversion to sorafenib significantly improves the OS in patients refractory to TACE therapy with intermediate-stage HCC. Administration of sorafenib is therefore recommended in such circumstances of TACE treatment failure.

Copyright $@ 2015$ S. Karger AG, Basel

\section{Introduction}

Hepatocellular carcinoma (HCC) is the third most common cause of cancer-related death worldwide and a growing cause of public health problems [1-3]. Because most patients are diagnosed with advanced stages of disease, only $30 \%$ of patients receive potentially curative therapies such as surgical resection $[4,5]$, transplantation $[6,7]$, or percutaneous ablation [8-10]. More specifically, the majority of patients with intermediate or advanced-stage HCCs usually undergo other palliative treatments such as transarterial chemoembolization (TACE) [11-13], hepatic arterial infusion chemotherapy (HAIC) [14], and systemic chemotherapy including molecular targeting agents [14-20].

TACE is currently the standard treatment for patients with multinodular HCC. Compared to hepatic resection, an advantage of TACE includes the preservation of liver function, which generally cannot be obtained after surgical treatment [11, 12, 17, 21-24]. Conversely, sorafenib, a molecular targeting agent, is currently the first-line agent for the treatment of unresectable HCC worldwide $[25,26]$. Indications for the use of sorafenib include the failure of response to TACE therapy, including extrahepatic spread and vascular invasion as well as the development of refractoriness to the treatment [14].

The Japan Society of Hepatology (JSH) and the Liver Cancer Study Group of Japan (LCSGJ) proposed a clear definition of 'refractoriness or failure to TACE' as described as follows: The first criterion applies to intrahepatic lesions, where refractoriness to TACE is defined as $\geq 2$ consecutive ineffective responses of treated tumors (viable lesions $>50 \%$ ) or $\geq 2$ consecutive progressive increases in total tumor count, despite a change of chemotherapeutic agent or selection of the feeding artery. For an ineffective response, it is recommended to reevaluate the patient using computed tomography (CT) or magnetic resonance imaging (MRI) at 1-3 months after a selective TACE treatment. Additional criteria of refractoriness to TACE include the continuous elevation of tumor marker levels immediately after the TACE, and new emergence of vascular invasion and extrahepatic spread after the procedure [23, 27].

An earlier study supports the robustness of the criteria regarding the TACE-refractoriness or failure as defined by the JSH and LCSGJ; overall survival (OS) was longer in patients treated with $<2$ consecutive ineffective TACE procedures than in patients who underwent $\geq 3$ consecutive TACE procedures before sorafenib administration [28]. Another study compared the efficacy of sorafenib and cisplatin in patients with HCC after refractoriness or failure of TACE and it reported that OS and time to progression (TTP) were longer in patients treated with sorafenib than in patients who underwent HAIC [29]. Ogasawara et al. compared the efficacy of sorafenib and continuous TACE treatment in patients with HCC, in addition to the refractoriness or failure of the TACE therapy; OS and TTP were longer in patients who were switched from TACE to sorafenib, than in patients who were treated with TACE alone [30]. In the present study, using large cohorts from our institution, we conducted a retrospective analysis comparing the OS of patients with HCC who were treated with sorafenib with those who underwent repeated TACE treatments during the TACE-refractory phase of their clinical course. 


\section{Materials and Methods}

\section{Patients}

The derivation dataset included 497 patients with HCC who were treated with TACE at Kinki University between 2008 and 2013. All patients satisfied the JSH diagnostic criteria for HCC [23]. HCC was diagnosed based on histological or radiological findings using contrast-enhanced CT (CE-CT) and/or dynamic MRI. The decision of providing TACE was made through discussion of multidisciplinary teams at our institution. Patients with intermediate-stage HCC who underwent repeated TACE treatments were retrospectively identified from our medical records; patients who were considered refractory to TACE were further selected and subdivided into two cohorts as follows: (1) patients who switched from TACE to sorafenib (sorafenib-group) and (2) those who continued on TACE despite being classified as refractory to the therapy (TACE-group).

The inclusion criteria for this study were: HCC diagnosed by histological examination or typical radiological findings (early enhancement followed by late wash-out on CE-CT or dynamic MRI), HCC cases not recommended for radiofrequency ablation or surgical resection, Barcelona Clinic Liver Cancer (BCLC) $[21,31]$ stage B (intermediate-stage), performance status (PS) of 0 or 1 , and Child-Pugh class A or B. The exclusion criteria were: concomitant antineoplastic treatment, BCLC stage C (advanced-stage), and having HAIC or systemic therapy after allocation of refractoriness to TACE therapy.

Clinicopathological variables including demographics, complete blood count, serum albumin level, C-reactive protein, alpha-fetoprotein (AFP), alanine aminotransferases and alkaline phosphatase, ChildPugh class, and BCLC $[21,31]$ prognostic scores were collected prior to each treatment.

\section{TACE Technique}

The right femoral artery was accessed with an 18-gauge Seldinger needle, and a 4-Fr sheath was then inserted. The celiac artery was selectively catheterized using a 4.2-Fr catheter. A 2.2-Fr microcatheter (Shirabe ${ }^{\circledR}$; Piolax, Yokohama, Japan) was advanced coaxially through the catheter into the common or proper hepatic artery. Rotational angiography was performed to evaluate the feeding vessels of identified HCCs. The tip of the catheter was selectively placed into feeding segmental and subsegmental arteries using the findings of selective hepatic angiography and/or tracking navigation imaging. Chemoembolization was performed using 60-120 mg of miriplatin (Miripla ${ }^{\circledR}$; Sumitomo Dainippon Pharma, Osaka, Japan), 20-50 mg of epirubicin (Epirubicin ${ }^{\circledR}$; Nippon Kayaku, Tokyo, Japan), or 50-100 mg of cisplatin (IA-call ${ }^{\circledR}$; Nippon Kayaku) emulsified with iodized oil (Lipiodol ${ }^{\circledR}$ Ultra-Fluid; Guerbet, Paris, France) and gelatin sponge particles (Gelpart ${ }^{\circledR}$; Nippon Kayaku or Gelfoam ${ }^{\circledR}$; Upjohn, Kalamazoo, MI, USA). The injection volume of the emulsion was determined according to tumor volumes $(\leq 8 \mathrm{ml})$. Drug-eluting-bead TACE (DEBTACE) or balloon-occluded TACE (B-TACE) was not utilized in this study.

\section{Definition of TACE Refractoriness}

A CT scan was performed within 3 months of the TACE procedure to evaluate the radiological response of the tumor(s) to the treatment. Follow-up CT or MRI was then performed every 3-4 months. In addition, AFP levels were evaluated every 2-3 months. The definition of refractoriness to TACE was based on the JSH Consensus Guidelines [23, 27]. The radiological response to TACE was evaluated using the initial CT or MRI within 3 months after TACE treatment. We determined the AFP level at baseline and at 2 months after the TACE treatment; a continuous elevation of the tumor marker was defined as an increase of $>20 \%$ from baseline. In the response assessment, only patients with a baseline AFP level of $>20 \mathrm{ng} / \mathrm{ml}$ were included. Patients received sorafenib therapy after evaluation of their refractoriness to TACE. However, some patients continued TACE therapy when they declined sorafenib therapy, and before sorafenib was authorized as a treatment for HCC.

\section{Treatment Strategy Using Sorafenib}

Sorafenib was administered orally at $400 \mathrm{mg}$, twice daily. Dose reduction of sorafenib and interruption of the therapy were allowed and depended on the type and severity of adverse events. Dose reduction and discontinuation of the drug were judged based on the information of the package insert. We continued sorafenib administration until the occurrence of intolerable toxicity or clear clinical disease progression was apparent. CE-CT or dynamic MRI was used to evaluate the tumor response; the radiological examinations were performed at baseline, at one month after the initiation of sorafenib and every 4-6 weeks thereafter. 
Statistical Analysis

Survival curves were estimated using the Kaplan-Meier method with death as the primary endpoint for the analysis of OS. Patients who did not meet the endpoint were censored at the time of the last followup visit. Survival rates were compared between the groups using the log-rank test, and categorical variables were compared using the chi-square test. Comparisons of categorical variables between the groups were conducted using Mann-Whitney U test. P values $<0.05$ were considered statistically significant. All analyses were performed using SAS ${ }^{\circledR}$ statistical software version 8.2 (SAS Institute, Cary, NC, USA) or the SPSS ${ }^{\circledR}$ Medical Pack for Windows version 10.0 (SPSS, Inc., Chicago, IL, USA).

\section{Results}

Characteristics of the Patients Enrolled in the Study

Among 497 who received TACE during their clinical course, 56 patients were diagnosed as refractory to the therapy. The baseline characteristics of the patients are summarized in table 1. Forty patients (71.4\%) were anti-hepatitis $\mathrm{C}$ virus antibody (HCV Ab) positive, four patients (7.1\%) were hepatitis B virus surface antigen (HBs-Ag) positive, and 12 (21.4\%) were negative for both $\mathrm{HCV} \mathrm{Ab}$ and HBs-Ag, respectively. Fifty-five (98.2\%) patients were asymptomatic, with a performance status of 0 . Forty-five patients $(80.4 \%)$ were classified as Child-Pugh A.

\section{Treatment after Classification as Refractory to TACE}

Of the patients classified as refractory to TACE, 32 were assigned to the sorafenib-group and 24 were assigned to the TACE-group. The characteristics of the patients in each group are summarized in table 2 . The TACE-group consisted of 14 men and 10 women with a median age of 77 years; 17 patients were classified as Child-Pugh A and seven were classified as Child-Pugh B. Among them, 17 (70.8\%) were positive for HCV Ab, and no patients were positive for HBs-Ag, and seven (29.2\%) patients were negative for both. Previous therapy of the patients included six hepatic resections, 11 local ablations, and two cases of cytotoxic systemic chemotherapy. Patients may have received more than one type of therapy. The sorafenib-group consisted of 24 men and eight women with a median age of 73 years; 28 patients were classified as Child-Pugh A, and four were classified as Child-Pugh B. Among these patients, 23 (71.9\%) were positive for HCV Ab, four (12.5\%) were positive for $\mathrm{HBs}-\mathrm{Ag}$, and five $(15.6 \%)$ were negative for both. Previous therapies included eight hepatic resections, 21 local ablations, and five cases of cytotoxic systemic chemotherapy. Patients may have received more than one type of therapy. There were no statistically significant differences in the patient characteristics between the two groups.

Of the patients enrolled in the study (32, sorafenib-group; 24, TACE-group), 38 died during the study period (19, sorafenib-group; 19, TACE-group), eight survived (5, sorafenibgroup; 3, TACE-group), and 10 were lost to follow-up (8, sorafenib-group; 2, TACE-group). The causes of death in the sorafenib-group were as follows: progression of the HCC (16 patients); and hepatic failure ( 3 patients). In the TACE-group the causes of death were: progression of the HCC (14 patients); and hepatic failure (5 patients).

In the sorafenib-group, 30 patients continued sorafenib until their disease progression. Two patients discontinued the treatment because of severe adverse events. Treatment discontinuation rates were $93.8 \%$. Dose reduction rates were $25 \%(8 / 32)$.

The median OS of the entire cohort was 10.27 months (95\% confidence interval [CI] 6.55-11.46 months). The median OS of the patients in the TACE- and sorafenib-group was 13.6 months (95\% CI 8.96-17.43 months) and 24.7 months (95\% CI 17.16-54.7 months), respectively. Pairwise comparisons verified a significantly longer OS in the sorafenib-group than in the TACE-group ( $\mathrm{p}=0.002$, log-rank test) (fig. 1). 
Table 1. Characteristics of HCC patients classified as refractory to TACE treatment

\begin{tabular}{|c|c|c|}
\hline & Number of patients $n=56$ & $(\%)$ \\
\hline Age, median (25-75\%) & $74(69-78)^{\mathrm{a}}$ & \\
\hline \multicolumn{3}{|l|}{ Sex } \\
\hline Male & 38 & -67.9 \\
\hline Female & 18 & -32.1 \\
\hline \multicolumn{3}{|l|}{ ECOG PS } \\
\hline 0 & 55 & -98.2 \\
\hline 1 & 1 & -1.8 \\
\hline \multicolumn{3}{|l|}{ Child-Pugh class } \\
\hline A & 45 & -80.4 \\
\hline $\mathrm{B}$ & 11 & -19.6 \\
\hline \multicolumn{3}{|l|}{ Virus status ${ }^{\mathrm{b}}$} \\
\hline HBV & 4 & -7.1 \\
\hline $\mathrm{HCV}$ & 40 & -71.4 \\
\hline Virus negative & 12 & -21.4 \\
\hline \multicolumn{3}{|l|}{ Previous therapy ${ }^{\mathrm{c}}$} \\
\hline Surgical resection & 14 & -25 \\
\hline Radiofrequency ablation & 32 & -57.1 \\
\hline Cytotoxic chemotherapy & 7 & -12.5 \\
\hline \multicolumn{3}{|l|}{ Biochemical analysis, median (25-75\%) } \\
\hline C-reactive protein, $\mathrm{mg} / \mathrm{dl}$ & $0.131(0.072-0.267)^{\mathrm{a}}$ & \\
\hline Alanine aminotransferase, IU/l & $41(33-57.5)^{\mathrm{a}}$ & \\
\hline Alkaline phosphatase, IU/l & $381(286-468)^{a}$ & \\
\hline AFP, ng/ml & $89(12.5-1057.5)^{\mathrm{a}}$ & \\
\hline Platelets, $10^{4} / \mu \mathrm{l}$ & $12.1(8.9-16.075)^{\mathrm{a}}$ & \\
\hline
\end{tabular}

ECOG=Eastern Cooperative Oncology Group; HBV=hepatitis B virus

a Dispersion variables are shown as median values (25-75\%). ${ }^{\mathrm{b}}$ Cases testing positive for HBs Ag were regarded as cases of HBV-related HCC, and cases testing positive for HCV Ab were regarded as cases of HCV-related HCC. 'Patients may have received more than one type of therapy.

Overall Comparison of the Child-Pugh Score after Classification as Refractory to TACE

In the TACE-group, the median number of TACE procedures that patients underwent was two (interquartile range $25-75 \%, 1-3$ procedures), after determination of refractoriness to TACE. In the sorafenib-group, the median duration of sorafenib administration was 124 days (interquartile range 25-75\%, 64-271 days). Both groups showed an increase in Child-Pugh scores at 3 months and 6 months after refractoriness to TACE, indicating deterioration in liver function. The median Child-Pugh scores after 3 months were 7 (25-75\% interquartile range, 6-7) in the TACE-group and 6 (25-75\% interquartile range, 5-7) in the sorafenib-group, with no significant differences seen between the two groups $(\mathrm{p}=0.051)$. However, the median Child-Pugh scores after 6 months were 7 (25-75\% interquartile range, 6.5-8.75) in the TACEgroup and 6 (25-75\% interquartile range, 5-6.5) in the sorafenib-group, with a significant difference between the two groups $(\mathrm{p}=0.005)$ (fig. $2 \mathrm{a})$. Further analyses were performed to 
Table 2. Comparison of patient characteristics among patients in the sorafenib conversion and TACE continuation groups

\begin{tabular}{|c|c|c|c|}
\hline & TACE group n=24 & Sorafenib group $n=32$ & $\mathrm{p}$ value \\
\hline Age, median (25-75\%) & $77(71.5-79.2)^{\mathrm{a}}$ & $73(68-77)^{a}$ & 0.088 \\
\hline \multicolumn{4}{|l|}{ Sex } \\
\hline Male & 14 & 24 & \\
\hline Female & 10 & 8 & 0.25 \\
\hline \multicolumn{4}{|l|}{ ECOG PS } \\
\hline 0 & 23 & 32 & \\
\hline 1 & 1 & 0 & 0.428 \\
\hline \multicolumn{4}{|l|}{ Child-Pugh class } \\
\hline A & 17 & 28 & \\
\hline B & 7 & 4 & 0.176 \\
\hline \multicolumn{4}{|l|}{ Virus status $^{\mathrm{b}}$} \\
\hline HBV & 0 & 4 & \\
\hline $\mathrm{HCV}$ & 17 & 23 & \\
\hline Virus negative & 7 & 5 & 0.086 \\
\hline \multicolumn{4}{|l|}{ Previous therapy, n (\%) } \\
\hline Surgical resection & $6(25)$ & $8(25)$ & 1 \\
\hline Radiofrequency ablation & $11(45.8)$ & $21(65.6)$ & 0.177 \\
\hline Cytotoxic chemotherapy & $2(8.3)$ & $5(15.6)$ & 0.686 \\
\hline \multicolumn{4}{|l|}{ Biochemical analysis, median (25-75\%) } \\
\hline C-reactive protein, $\mathrm{mg} / \mathrm{dl}$ & $0.12(0.05-0.16)^{\mathrm{a}}$ & $0.18(0.09-0.48)^{\mathrm{a}}$ & 0.071 \\
\hline Alanine aminotransferase, IU/l & $37(30-51)^{a}$ & $48(35-60)^{\text {a }}$ & 0.134 \\
\hline Alkaline phosphatase, IU/l & $391.5(293-530)^{\mathrm{a}}$ & $381(279-463.2)^{\mathrm{a}}$ & 0.524 \\
\hline $\mathrm{AFP}, \mathrm{ng} / \mathrm{ml}$ & $144(11.5-540)^{a}$ & $72(14-1977)^{a}$ & 0.812 \\
\hline Platelets, $10^{4} / \mu \mathrm{l}$ & $11.8(9.1-15.1)^{\mathrm{a}}$ & $12.5(8.5-17.7)^{\mathrm{a}}$ & 0.625 \\
\hline
\end{tabular}

${ }^{a}$ Dispersion variables are shown as median values (25-75\%). ${ }^{\mathrm{b}}$ Patients testing positive for HBV surface antigen were regarded as cases of HBV-related HCC, and patients testing positive for HCV antibody were regarded as cases of HCV-related HCC. 'Patients may have received more than one type of therapy.

examine the changes in serum albumin levels, serum bilirubin levels, and prothrombin time (PT), because these items as part of the Child-Pugh score had shown greater clinical changes in the study cohort. Serum bilirubin levels were not significantly different between the two groups after 3 months $(\mathrm{p}=0.28)$ or 6 months ( $\mathrm{p}=0.067)$. After 3 months, the median bilirubin level was $0.9 \mathrm{mg} / \mathrm{dl}$ (25-75\% interquartile range, $0.5-1.7 \mathrm{mg} / \mathrm{dl})$ in the TACE-group and $0.8 \mathrm{mg} / \mathrm{dl}(25-75 \%$ interquartile range, $0.5-1.2 \mathrm{mg} / \mathrm{dl})$ in the sorafenib-group. After 6 months, the median bilirubin level was $1.25 \mathrm{mg} / \mathrm{dl}$ (25-75\% interquartile range, $0.62-2.45$ $\mathrm{mg} / \mathrm{dl})$ in the TACE-group and $0.7 \mathrm{mg} / \mathrm{dl}(25-75 \%$ interquartile range, $0.5-1.25 \mathrm{mg} / \mathrm{dl})$ in the sorafenib-group (fig. 2b). In contrast, a significant difference of serum albumin levels were observed at 6 months $(p=0.043)$ but not at 3 months $(p=0.14)$. After 3 months, the median albumin level was $3.4 \mathrm{~g} / \mathrm{dl}(25-75 \%$ interquartile range, $3.2-3.6 \mathrm{~g} / \mathrm{dl})$ in the TACE-

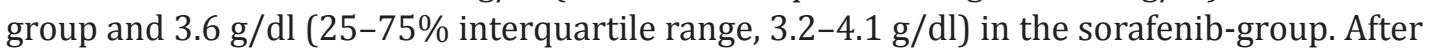




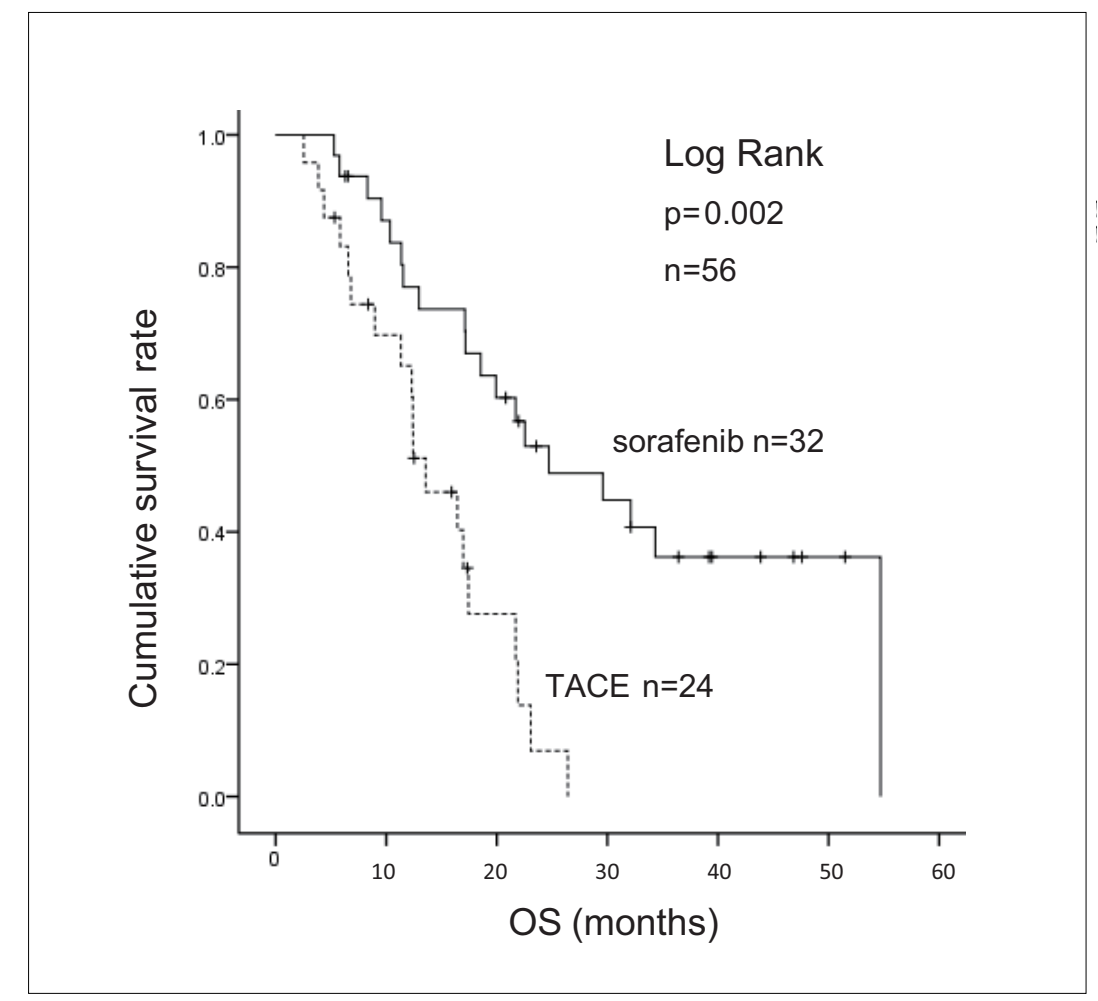

Fig. 1 Comparison of OS between TACE and sorafenib among patients deemed refractory to TACE. Kaplan-Meier curves of the OS of 56 patients who underwent treatment with continued TACE or conversion to sorafenib after being deemed refractory to TACE. The median OS of patients assigned to the TACE and sorafenib groups was 13.6 and 24.7 months, respectively, after being deemed refractory to TACE ( $\mathrm{p}=0.002$ by log-rank test).

6 months, the median albumin level was $3.15 \mathrm{~g} / \mathrm{dl}$ (25-75\% interquartile range, $2.8-3.5 \mathrm{~g} / \mathrm{dl}$ ) in the TACE-group and $3.6 \mathrm{~g} / \mathrm{dl}$ (25-75\% interquartile range, $3.1-4.15 \mathrm{~g} / \mathrm{dl})$ in the sorafenibgroup (fig. 2c). Similarly, the difference in PT between the two groups was also significant at 6 months ( $\mathrm{p}=0.0041)$ but not at 3 months ( $\mathrm{p}=0.069)$. After 3 months, the median PT was $78.15 \%(25-75 \%$ interquartile range, $64.47-87.3 \%)$ in the TACE-group and $82.15 \%(25-75 \%$ interquartile range, 76.57-89.62\%) in the sorafenib-group. After 6 months, the median PT was $72.25 \%$ (25-75\% interquartile range, 63.67-85.2\%) in the TACE-group and $86.5 \%$ (25$75 \%$ interquartile range, 77.05-92.8\%) in the sorafenib-group, respectively (fig. $2 \mathrm{~d}$ ).

\section{Discussion}

Patients with vascular invasion or extrahepatic metastasis, or with insufficient response to TACE are good candidates for sorafenib. The efficacy of sorafenib in cases refractory to TACE have been reported previously $[29,30]$. Ogasawara et al. found that time to disease progression and OS were prolonged by switching the treatment to sorafenib in patients with intermediate-stage HCC, if TACE refractoriness was confirmed [30]. The present study also demonstrates that OS is longer in the sorafenib-group than in the TACE-group in patients with HCC who are deemed refectory to TACE, using the definition of TACE failure or refractoriness proposed by the JSH and LCSGJ. 


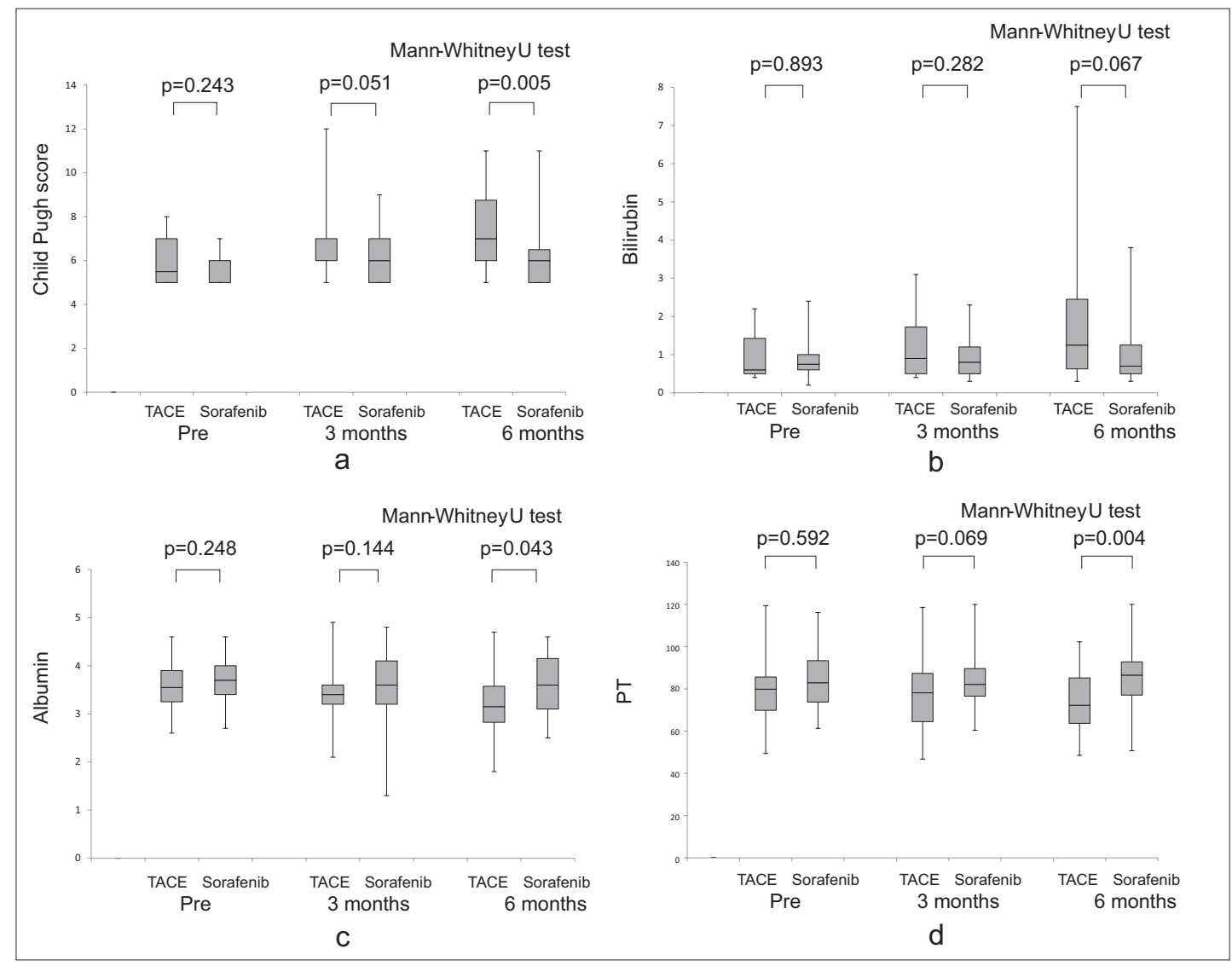

Fig. 2 Overall comparison of Child-Pugh score between the sorafenib conversion group and the TACE continuation group over time.

a A significant difference was noted in Child-Pugh score 6 months after patients were deemed refractory to TACE ( $p=0.005$ by Mann-Whitney U test). Each bar represents $25^{\text {th }}-75^{\text {th }}$ percentile. The dashed line indicates the alteration in the median value of the TACE group and solid line represents the sorafenib group. b No significant difference was noted in the plasma bilirubin at 3 or 6 months after patients were deemed refractory to TACE. c A significant difference was noted in plasma albumin at 6 months after patients were deemed refractory to TACE ( $p=0.043$ by Mann-Whitney U test). $\mathbf{d}$ A significant difference was noted in the PT at 6 months after patients were deemed refractory to TACE $(p=0.0041$ by MannWhitney U test).

The Sorafenib HCC Assessment Randomized Protocol (SHARP) and the Asia-Pacific trials included $54(18 \%)$ and $7(4.7 \%)$ patients with intermediate-stage HCC, who would typically qualify for TACE as a first-line treatment according to the JSH and LCSGJ guidelines $[25,26]$. Notably, $86(29 \%)$ and $61(40.7 \%)$ of the patients in these trials underwent TACE treatment as a preceding therapy $[25,26,32]$. The Asia-Pacific trial also evaluated the OS of sorafenib-treated and placebo groups among the patients who underwent preceding TACE therapy; the OS was 7.3 months (95\% CI, 5.2-9.5) in the sorafenib-group and 5.1 months (95\% CI, 4.1-11.3) in the placebo-group, with a hazard ratio of 0.84 (95\% CI, 0.52-1.36), while the TTP was 2.7 months (95\% CI, 1.5-2.8) in the sorafenib-group and 1.3 months (95\% CI, 1.3-1.4) in the placebo-group, with a hazard ratio of 0.57 (95\% CI, 0.36-0.90), respectively [32]. These findings are quite similar to our study results, demonstrating the effectiveness of sorafenib even in the patients with a previous history of TACE, which has been recommended as a conventional first-line treatment. 
Takayasu et al. [33] reported that independent prognostic factors of survival in patients who underwent TACE included the degree of hepatic damage, the tumor stage, and the serum AFP level. However, no studies have confirmed that repeated TACE may lead to deteriorations of liver function. Our results indicate that the increase in Child-Pugh score is greater in the TACE-group compared to the sorafenib-group. Given that the subjects of the present study developed refractoriness to TACE, the deterioration in liver function may have been related to the shorter interval of TACE procedures in the advanced clinical stage or progression of the tumors. Among the items of the Child-Pugh score, a decrease of the serum albumin and PT levels was prominent, suggesting an impact of TACE on protein synthesis. Although, the reduction of OS in patients refractory to TACE may be principally attributable to the progression of the primary tumors, it is possible that repeated TACE over a shorter interval might, at least partially, contribute to the reduction in OS.

In the present study, all subjects underwent conventional TACE (CTACE). However, no studies have examined whether recently developed TACE procedures, such as DEB-TACE or B-TACE are effective in patients with HCC who are refractory to TACE. Considering that all subjects in the present study underwent CTACE, further studies are required to determine the efficacy of DEB-TACE or B-TACE in such cases.

Although we analyzed considerable numbers of patients, the retrospective design of the study may have led to selection bias of the patients. There were 10 patients lost to follow up in this study, which may also have led to bias. To further validate the results of this study, we are currently conducting a prospective multicenter study to confirm the significance of sorafenib in patients with HCC who have failed or who are refractory to TACE treatment.

In conclusion, our data strongly support the fact that conversion to sorafenib improves outcome as evidenced by an increase in OS in patients refractory to TACE with intermediatestage HCC. Repeated TACE may induce resistance to chemotherapy, which in turn may increase the risk of tumor recurrence and metastasis. More importantly, it may induce deterioration of liver function, which is critical for the safely treatment with sorafenib. Thus, to improve the survival of patients with HCC refractory to TACE, it is important to switch the treatment from TACE to sorafenib even if the tumor is still in the intermediate stage.

\section{Disclosure Statement}

The authors declare no conflict of interest.

\section{References}

1 El-Serag HB, Rudolph KL: Hepatocellular carcinoma: epidemiology and molecular carcinogenesis. Gastroenterology 2007;132:2557-2576.

2 Parkin DM: Global cancer statistics in the year 2000. Lancet Oncol 2001;2:533-543.

3 Kim Y, Han KH: Epidemiology and surveillance of hepatocellular carcinoma. Liver Cancer 2012;1:2-14.

4 Belghiti J, Fuks D: Liver resection and transplantation in hepatocellular carcinoma. Liver Cancer 2012;1:71-82.

5 Shindoh J, D Tzeng CW, Vauthey JN: Portal vein embolization for hepatocellular carcinoma. Liver Cancer 2012;1:159-167.

6 Lee Cheah Y, K H Chow P: Liver transplantation for hepatocellular carcinoma: an appraisal of current controversies. Liver Cancer 2012;1:183-189.

7 Chan SC: Liver transplantation for hepatocellular carcinoma. Liver Cancer 2013;2:338-344.

8 Bruix J, Llovet JM: Prognostic prediction and treatment strategy in hepatocellular carcinoma. Hepatology 2002;35:519-524.

9 Park KW, Park JW, Choi JI, Kim TH, Kim SH, Park HS, Lee WJ, Park SJ, Hong EK, Kim CM: Survival analysis of 904 patients with hepatocellular carcinoma in a hepatitis B virus-endemic area. J Gastroenterol Hepatol 2008;23:467-473. 
10 Lin SM: Local ablation for hepatocellular carcinoma in taiwan. Liver Cancer 2013;2:73-83.

11 Bruix J, Sala M, Llovet JM: Chemoembolization for hepatocellular carcinoma. Gastroenterology 2004;127(Suppl 1):S179-S188.

12 Lencioni R: Chemoembolization in patients with hepatocellular carcinoma. Liver Cancer 2012;1:41-50.

13 Nishikawa H, Kita R, Kimura T, Osaki Y: Transcatheter arterial embolic therapies for hepatocellular carcinoma: a literature review. Anticancer Res 2014;34:6877-6886.

14 Kudo M: Treatment of advanced hepatocellular carcinoma with emphasis on hepatic arterial infusion chemotherapy and molecular targeted therapy. Liver Cancer 2012;1:62-70.

15 Ando E, Tanaka M, Yamashita F, Kuromatsu R, Yutani S, Fukumori K, Sumie S, Yano Y, Okuda K, Sata M: Hepatic arterial infusion chemotherapy for advanced hepatocellular carcinoma with portal vein tumor thrombosis: analysis of 48 cases. Cancer 2002;95:588-595.

16 Obi S, Yoshida H, Toune R, Unuma T, Kanda M, Sato S, Tateishi R, Teratani T, Shiina S, Omata M: Combination therapy of intraarterial 5-fluorouracil and systemic interferon-alpha for advanced hepatocellular carcinoma with portal venous invasion. Cancer 2006;106:1990-1997.

17 Lin S, Hoffmann K, Schemmer P: Treatment of hepatocellular carcinoma: a systematic review. Liver Cancer 2012;1:144-158.

18 Peck-Radosavljevic M: Drug therapy for advanced-stage liver cancer. Liver Cancer 2014;3:125-131.

19 Benson AB 3rd, Abrams TA, Ben-Josef E, Bloomston PM, Botha JF, Clary BM, Covey A, Curley SA, D’Angelica MI, Davila R, Ensminger WD, Gibbs JF, Laheru D, Malafa MP, Marrero J, Meranze SG, Mulvihill SJ, Park JO, Posey JA, Sachdev J, Salem R, Sigurdson ER, Sofocleous C, Vauthey JN, Venook AP, Goff LW, Yen Y, Zhu AX: NCCN clinical practice guidelines in oncology: hepatobiliary cancers. J Natl Compr Canc Netw 2009;7:350-391.

20 Takeda H, Nishikawa H, Osaki Y, Tsuchiya K, Joko K, Ogawa C, Taniguchi H, Orito E, Uchida Y, Izumi N, Japanese Red Cross Liver Study Group: Clinical features associated with radiological response to sorafenib in unresectable hepatocellular carcinoma: a large multicenter study in Japan. Liver Int 2015;35:1581-1589.

21 Bruix J, Sherman, M American Association for the Study of Liver Diseases: Management of hepatocellular carcinoma: an update. Hepatology 2011;53:1020-1022.

22 European Association For The Study Of The Liver, European Organisation For Research And Treatment Of Cancer: EASL-EORTC clinical practice guidelines: management of hepatocellular carcinoma. J Hepatol 2012;56:908-943.

23 Kudo M, Izumi N, Kokudo N, Matsui O, Sakamoto M, Nakashima O, Kojiro M, Makuuchi M, HCC Expert Panel of Japan Society of Hepatology: Management of hepatocellular carcinoma in Japan: Consensus-Based Clinical Practice Guidelines proposed by the Japan Society of Hepatology (JSH) 2010 updated version. Dig Dis 2011;29:339-364.

24 Lencioni R: Chemoembolization for hepatocellular carcinoma. Semin Oncol 2012;39:503-509.

25 Llovet JM, Ricci S, Mazzaferro V, Hilgard P, Gane E, Blanc JF, de Oliveira AC, Santoro A, Raoul JL, Forner A, Schwartz M, Porta C, Zeuzem S, Bolondi L, Greten TF, Galle PR, Seitz JF, Borbath I, Häussinger D, Giannaris T, Shan M, Moscovici M, Voliotis D, Bruix J, SHARP Investigators Study Group: Sorafenib in advanced hepatocellular carcinoma. N Engl J Med 2008;359:378-390.

26 Cheng AL, Kang YK, Chen Z, Tsao CJ, Qin S, Kim JS, Luo R, Feng J, Ye S, Yang TS, Xu J, Sun Y, Liang H, Liu J, Wang J, Tak WY, Pan H, Burock K, Zou J, Voliotis D, Guan Z: Efficacy and safety of sorafenib in patients in the Asia-Pacific region with advanced hepatocellular carcinoma: a phase III randomised, double-blind, placebo-controlled trial. Lancet Oncol 2009;10:25-34.

27 Kudo M, Matsui O, Izumi N, Kadoya M, Okusaka T, Miyayama S, Yamakado K, Tsuchiya K, Ueshima K, Hiraoka A, Ikeda M, Ogasawara S, Yamashita T, Minami T, Liver Cancer Study Group of Japan: Transarterial chemoembolization failure/refractoriness: JSH-LCSGJ criteria 2014 update. Oncology 2014;87(Suppl 1):22-31.

28 Arizumi T, Ueshima K, Chishina H, Kono M, Takita M, Kitai S, Inoue T, Yada N, Hagiwara S, Minami Y, Sakurai T, Nishida N, Kudo M: Validation of the criteria of transcatheter arterial chemoembolization failure or refractoriness in patients with advanced hepatocellular carcinoma proposed by the LCSGJ. Oncology 2014;87(Suppl 1):32-36.

29 Ikeda M, Mitsunaga S, Shimizu S, Ohno I, Takahashi H, Okuyama H, Kuwahara A, Kondo S, Morizane C, Ueno H, Satake M, Arai Y, Okusaka T: Efficacy of sorafenib in patients with hepatocellular carcinoma refractory to transcatheter arterial chemoembolization. J Gastroenterol 2014;49:932-940.

30 Ogasawara S, Chiba T, Ooka Y, Kanogawa N, Motoyama T, Suzuki E, Tawada A, Kanai F, Yoshikawa M, Yokosuka 0: Efficacy of sorafenib in intermediate-stage hepatocellular carcinoma patients refractory to transarterial chemoembolization. Oncology 2014;87:330-341.

31 Llovet JM, Brú C, Bruix J: Prognosis of hepatocellular carcinoma: the BCLC staging classification. Semin Liver Dis 1999;19:329-338.

32 Cheng AL, Guan Z, Chen Z, Tsao CJ, Qin S, Kim JS, Yang TS, Tak WY, Pan H, Yu S, Xu J, Fang F, Zou J, Lentini G, Voliotis D, Kang YK: Efficacy and safety of sorafenib in patients with advanced hepatocellular carcinoma according to baseline status: subset analyses of the phase III Sorafenib Asia-Pacific trial. Eur J Cancer 2012;48:1452-1465.

33 Takayasu K, Arii S, Ikai I, Omata M, Okita K, Ichida T, Matsuyama Y, Nakanuma Y, Kojiro M, Makuuchi M, Yamaoka Y, Liver Cancer Study Group of Japan: Prospective cohort study of transarterial chemoembolization for unresectable hepatocellular carcinoma in 8510 patients. Gastroenterology 2006;131:461-469. 